\title{
Ocular surface disease in intensive care unit patients
}

\begin{abstract}
Purpose To assess the prevalence and natural history of ocular surface disease in patients in an intensive care unit (ICU) and to identify any predisposing factors in order to improve eye care management protocols.

Methods The eyes of all patients admitted to an ICU between February and May 1998 were examined at least weekly. The eyelid position, presence of conjunctival oedema, degree of keratopathy and sedation score were documented at every assessment.

Results Twenty-six patients in an ICU were followed throughout their stay, which ranged from 3 days to 10 weeks. Eleven patients $(42 \%)$ had some degree of keratopathy, which was detected in the majority in the first week of their stay. The presence of ocular surface disease was closely correlated with the degree of lagophthalmos, which in turn is closely related to the depth of sedation or paralysis. Conclusions In this first ever longitudinal study it was found that assessment of lid position in ICU patients is the single most important observation to be carried out. A management algorithm derived from this evidence is based on daily observation and selective lid taping and shows encouraging early results.
\end{abstract}

Key words Conjunctival oedema, Intensive care unit, Lagophthalmos, Sedation score

Patients in an intensive care unit (ICU) are potentially vulnerable to ocular surface disease, which at worst can lead to microbial keratitis. ${ }^{1-7}$ Preventative eye care measures are often overlooked since the medical and nursing teams in the ICU are mainly concerned with stabilisation of the cardiovascular, respiratory and neurological status, and patients may be unable to convey ocular symptoms. In a prospective analysis of 50 randomly selected ICU patients Hernandez and Mannis ${ }^{8}$ found superficial keratopathy in $40 \%$. Imanaka et al. ${ }^{9}$ have quoted a $60 \%$ prevalence of keratopathy in a prospective analysis of subjects who had been heavily sedated or paralysed for more than $48 \mathrm{~h}$. In both these studies ICU patients were examined on one occasion only.
FRANCO MERCIECA, PALANISAMY SURESH, ALAN MORTON, ANDREW TULLO
We have undertaken a prospective longitudinal study to assess the natural history of ocular surface disease in the ICU and to identify any predisposing factors that could be used to derive an eye care management protocol based on evidence.

\section{Materials and methods}

The eyes of all patients admitted between February and May of 1998 to the ICU at the Manchester Royal Infirmary (maximum bed capacity of 10) were assessed at least weekly by a single observer (F.M.). Informed consent was obtained from patients or relatives. Patients who had facial or eye injuries or who died within 2 days were excluded from this study.

The position of the eyelids with respect to the globe was noted with the use of a bright handheld pen torch. The eyelid position, conjunctival oedema and keratopathy were categorised as shown in Tables 1-3. In each patient these observations were based on the findings in the eye with more keratopathy or, in its absence, the degree of lagophthalmos. There were only two instances where this had to be used since all other patients had symmetrical findngs. The blink reflex was not tested because of concern that repeated and even single trauma to the cornea might result in significant ocular morbidity.

The nursing staff were asked about the details of the current eye care including the use of any eye medications or artificial eyelid closure. The following information was documented: (a) reason for admission, (b) date of admission, (c) demographics, (d) presence of intubation and the method of intubation, viz.

Table 1. Eyelid position grading

\begin{tabular}{ll}
\hline Grade 1 & Lids apposed \\
Grade 2 & Conjunctiva exposed \\
Grade 3 & Limbus exposed
\end{tabular}

Grade 3 Limbus exposed

Table 2. Conjunctival oedema grading

\begin{tabular}{|c|c|}
\hline Grade 0 & Absent \\
\hline Grade 1 & $\begin{array}{l}\text { Conjunctival oedema without dellen } \\
\text { formation }\end{array}$ \\
\hline Grade 2 & $\begin{array}{l}\text { Conjunctival oedema with dellen } \\
\text { formation }\end{array}$ \\
\hline
\end{tabular}

F. Mercieca

P. Suresh

A. Tullo

Manchester Royal Eye

Hospital

Manchester M13 9WH, UK

A. Morton

Department of Anaesthesia Manchester Royal Infirmary Manchester M13 9WH, UK

Mr A.B. Tullo

Manchester Royal Eye

Hospital

Oxford Road

Manchester M13 9WH, UK

Tel: $+44(0) 1612765522$

Fax: +44 (0)161272 6618

e-mail: atullo@central.

cmht.nwest.nhs.uk

Received: 20 July 1998

Accepted in revised form: 30 November 1998 
Table 3. Ocular surface disease category

i Punctate epithelial erosions (PEEs) involving the inferior third of the cornea

ii PEEs involving more than the inferior third of the corneal surface

iii Macroepithelial defect (MED)

iv Stromal whitening in the presence of epithelial defect (SWED)

$\mathrm{v}$ Stromal scar

vi Microbial keratitis (MK) ${ }^{\mathrm{a}}$

vii Other ocular surface disorders

${ }^{a}$ Microbial keratitis was defined as the presence of an epithelial defect with stromal infiltrate and a positive bacterial culture.

endotracheal tube or tracheostomy, (e) method of artificial feeding, i.e. nasogastric tube, via jejunostomy or parenteral access, (f) sedation scale and $(\mathrm{g})$ use of muscle relaxants.

Most ICU patients were sedated to some degree. The depth of sedation was assessed according to the sedation scale shown in Table 4 and the sedation score can be classified as shown in Table $5 .^{10}$ After each assessment the ICU medical team was informed of any recorded changes to the eye care and appropriate follow-up was arranged.

\section{Results}

Over the 4-month study period 26 patients were included. Fourteen patients were examined between 2 and 6 times during their stay in the ICU and all within $48 \mathrm{~h}$ of admission for the first time. The total number of assessments was 57 .

The age of patients ranged between 21 and 79 years. There was a male preponderance of $4: 1$. The duration of hospitalisation in the ICU varied between 2 and 72 days.

Table 4. Sedation scale

\begin{tabular}{lc}
\hline 1. Eyes open (lid movement) & \\
Spontaneously & 4 \\
To speech & 3 \\
To pain & 2 \\
$\quad$ None & 1 \\
2. Response to nursing procedures & \\
Obeys commands & 4 \\
Purposeful movement & 3 \\
Non-purposeful movement & 2 \\
None & 1 \\
3. Cough & \\
Spontaneous strong & \\
Spontaneous weak & 4 \\
On suction only & 3 \\
None & 2 \\
4. Respirations & 1 \\
Extubated & \\
Spontaneous intubated & \\
SIMV triggered & 5 \\
Against ventilator & 4 \\
No respiratory effort & 3 \\
5. Loading for spontaneous communication & 2 \\
\hline
\end{tabular}

SIMV, spontaneous intermittent mandatory ventilation.
Table 5. Sedation score

\begin{tabular}{lc}
\hline Awake & $17-19$ \\
Asleep & $15-17$ \\
Light sedation & $12-14$ \\
Moderate sedation & $8-11$ \\
Deep sedation & $5-7$ \\
Anaesthetised & 4 \\
\hline
\end{tabular}

There was no obvious correlation of the degree of keratopathy with the age, sex, diagnosis or method of artificial feeding. Only two patients had asymmetrical findings, one having worse signs on the right and the other on the left.

All patients were on 'routine eye care', which consisted of cleaning eyelids with gauze soaked in normal saline or sterile water every $2-6 \mathrm{~h}$. Two patients were also given chloramphenicol ointment. Treatment was altered by adding topical lubricants or antibiotics, with or without lid taping, in 9 patients.

Among the 26 patients, 11 (42\%) were found to have some degree of keratopathy at some point during their stay in the ICU. Seven of the affected patients had inferior punctate epithelial erosions of variable degree that resolved on treatment without any identifiable longterm sequelae. All these patients had limbal or corneal exposure and were under moderate to deep sedation.

Three were found to have a macroepithelial defect in the inferior third of the cornea that progressed to an anterior stromal scar despite treatment. All these 3 patients had corneal exposure with grade 2 chemosis and were either on muscle relaxants or deep sedation at the time of the corneal complications. One of these patients developed severe exposure keratopathy during prone ventilation. Another affected patient was found to have diffuse superficial keratopathy under closed lids. This patient was off sedation or muscle relaxants altogether but was deeply comatose following a massive intracerebral haemorrhage and he died 2 days later.

Twelve patients $(46 \%)$ retained full lid apposition throughout their stay in the ICU. Four patients had conjunctival exposure only and did not develop keratopathy. The correlation between the lid position and presence of keratopathy is shown in Fig. 1.

The sedation score of patients varied throughout their stay in the ICU. Generally the patients were deeply sedated initially and gradually weaned as the clinical status improved. As shown in Fig. 2 the degree of keratopathy was closely related to the depth of sedation or muscle relaxants. However, a fair proportion (25\%) of the patients who were under deep sedation showed no evidence of ocular surface disease. These patients maintained good eyelid closure.

Conjunctival oedema had a high incidence of $80 \%$ (21 patients). Six were at grade 2 and had concomitant keratopathy. Almost all those who had lid malposition also had some degree of chemosis (92\%), as shown in Fig. 3. However, chemosis was also noted in 6 patients who retained full lid apposition throughout their stay in the ICU. 


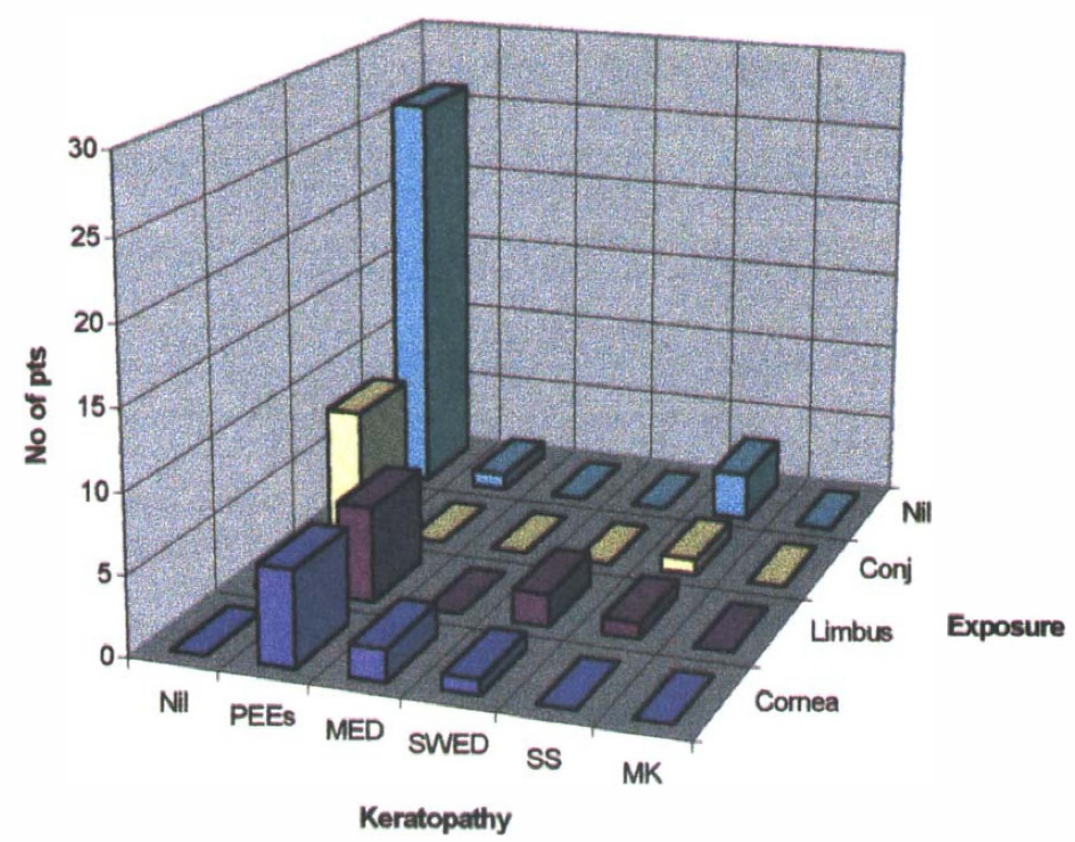

Fig. 1. The correlation between lid malposition and degree of keratopathy. Ocular surface disease occurred almost always in the presence of lagophthalmos. PEEs, punctate epithelial erosions; MED, macroepithelial defect; SWED, stromal whitening and epithelial defect; MK, microbial keratitis. NB: Since most patients were assessed periodically for several weeks, each assessment is represented separately.

The group of patients who had an endotracheal tube intubation (ETT) had a higher incidence of lid malposition ( $70 \%$ vs $24 \%$ ), keratopathy ( $49 \%$ vs $20 \%$ ) and conjunctival chemosis ( $79 \%$ vs $38 \%$ ) compared with the tracheostomy group. However, the ETT group also were under deep sedation more often than the tracheostomy group ( $55 \%$ vs $10 \%$ respectively).

Ocular surface disease was highly prevalent in the early stages of hospitalisation (Fig. 4). Nine of the 11 patients $(82 \%)$ who developed keratopathy were noted to have the signs in their first week of stay.

\section{Discussion}

In normal circumstances the eye is well equipped with natural defence mechanisms to protect the ocular surface. ${ }^{11}$ The lids provide a physical barrier to trauma and desiccation and prevent adherence of organisms to the ocular surface. The tear film provides a moist oxygenated environment for epithelial cells, lubricating the corneal surface while they dilute and remove noxious stimuli. Tears are bactericidal since they contain several antimicrobial substances in the form of lysozyme,

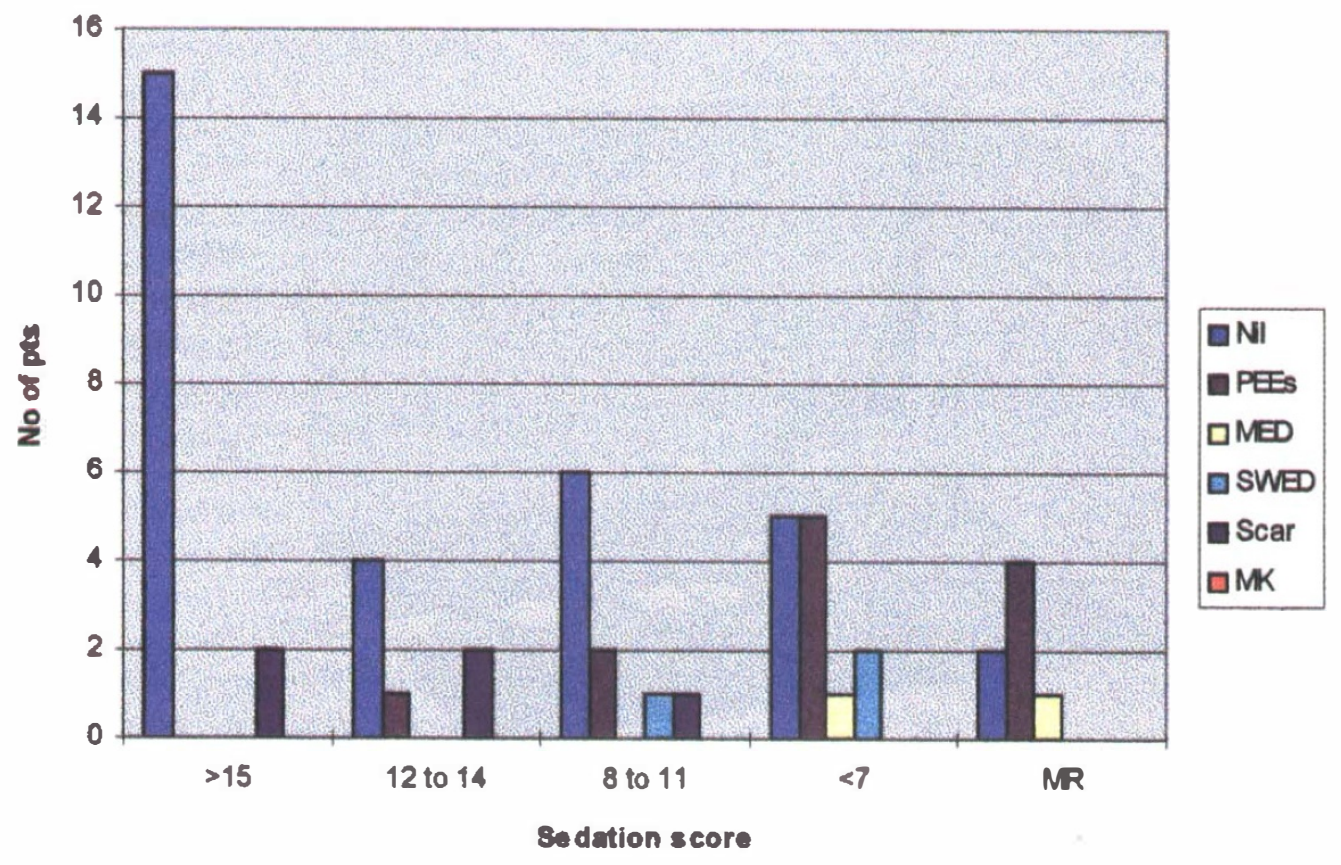

Fig. 2. Correlation between degree of sedation and keratopathy. Ocular surface disease is closely correlated with heavy sedation or muscle relaxants (MR). Abbreviations as in Fig. 1. NB: This represents only 54 sets of data as the remaining 3 patients were not on any sedation. 


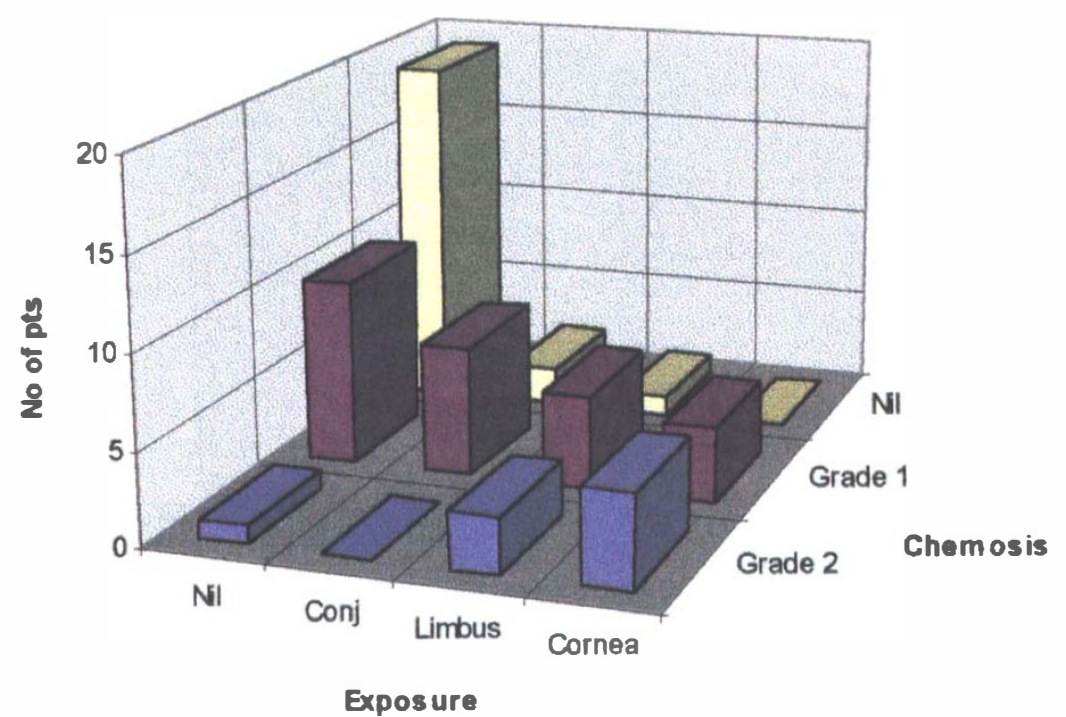

Fig. 3. Correlation between lid malposition and grade of chemosis. Most patients who had lagophthalmos also had conjunctival chemosis.

B-lysin, lactoferrin and immunoglobulins. The blink reflex of the lids leads to distribution of the tears over the whole of the ocular surface. The presence of good lid function and tear film maintain an intact, healthy corneal epithelium.

Eyelid closure during sleep does not occur solely due to relaxation of the periorbital muscles that otherwise keep the eye open, but is rather due to an active process involving contraction of orbicularis oculi with concomitant inhibition of the levator palpebrae superioris. ${ }^{12}$ Therefore it is easy to understand how muscle relaxants that abolish the tonic contraction of the orbicularis allow the eyelids to take a position dependent on the balance of passive forces. Sedation also decreases the blink reflex, which increases the propensity towards exposure keratopathy. The commonest scenario in the ICU is incomplete lid closure due to heavy sedation or muscle relaxants that, unless treated, inevitably leads to exposure keratopathy. It is of interest to note that crack cocaine users are also susceptible to sterile epithelial defects and infectious ulcers, perhaps due to a combination of factors including drug-induced stupor and lagophthalmos. ${ }^{13}$ Indeed, lagophthalmos has been well documented to occur during sleep leading to exposure sequelae in otherwise normal subjects. ${ }^{14}$

The study was observational not interventional and was not designed to change practice during its course. However, for ethical reasons ophthalmological advice was given when requested or felt to be appropriate.

From our study it is clear that the position of the eyelids is pivotal in the development of ocular surface disease. Seventy-five per cent of patients under heavy sedation or muscle relaxants were found to have lagophthalmos. This suggests that lagophthalmos in ICU

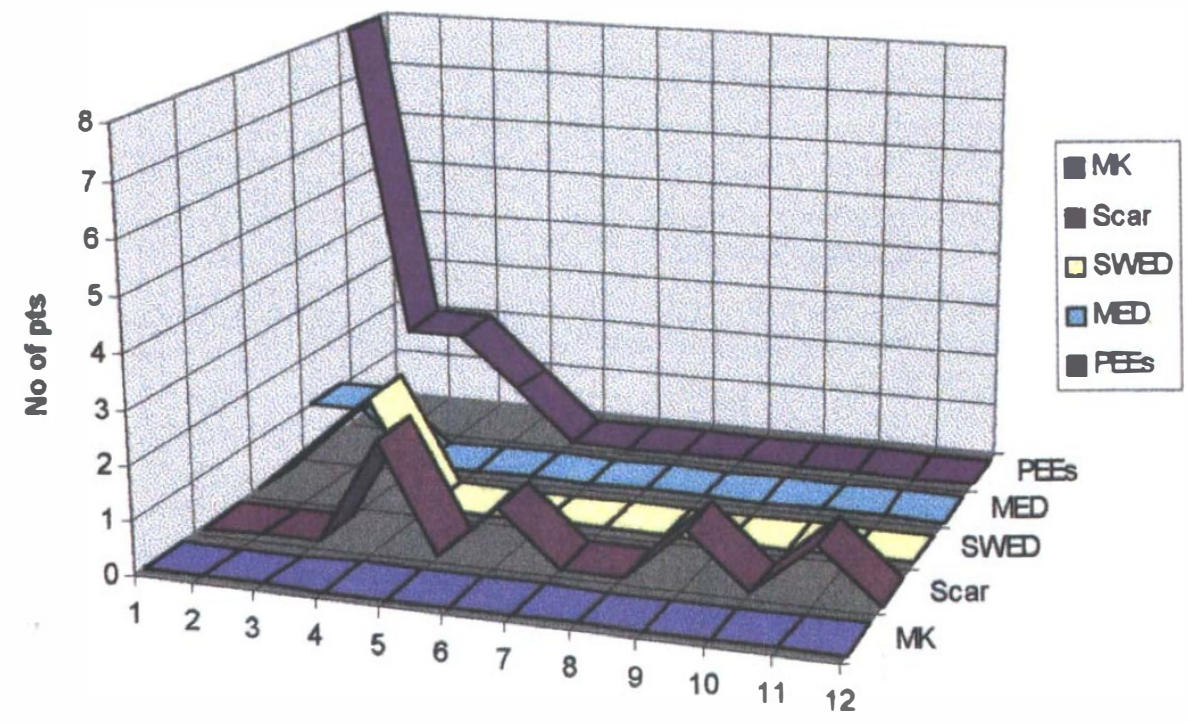

Duration of stay on ICU (weeks)

Fig. 4. Correlation between duration of stay on the ICU and prevalence of keratopathy. Ocular surface disease is most prevalent in the first 2 weeks of ICU stay. Abbreviations as in Fig. 1. 
patients is not only dependent on degree of sedation but may arise from an inherent ability to close the lids. From our experience in this study, proper examination of lid apposition is not always carried out. This is due to the eyelashes obscuring the lid gape, especially on the medial aspect. Meticulous assessment of lid apposition is paramount and we feel is best done with a light source in line with the eyelashes.

The ocular surface disease that we observed in these patients began as punctate epithelial erosions in the inferior third of the cornea and, unless treated, progressed into macroepithelial defects. In 3 patients this then developed into an anterior stromal whitening in the presence of an epithelial defect later healing to leave a stromal scar. The anterior stromal whitening is partly due to stromal desiccation but is also a feature of microbial keratitis.

Conjunctival oedema, which has also been termed 'ventilator eye', ${ }^{15}$ was very common. This is attributed to the high jugular venous pressure which in turn leads to fluid leakage in the periocular tissues. Raised venous pressure is associated with positive pressure ventilation, tight endotracheal tube tape ${ }^{15}$ and metabolic causes such as hypoproteinaemia. In our study all patients who had lagophthalmos exhibited some degree of chemosis. This suggests that the chemosis may be secondary to exposure itself or that in some patients it may be a contributing or prime factor leading to lagophthalmos.

We found a higher incidence of chemosis in those patients with an ETT compared with those with a tracheostomy. This may be partly attributed to the tight tape used to keep the ETT in place. However, the ETT group also had a higher incidence of lagophthalmos and consequent keratopathy and this was directly related to deeper sedation than in the tracheostomy group. The general trend is for patients in the ICU to have an ETT in the initial phase and only to have a tracheostomy when they are being weaned off sedation and if a relatively long stay is anticipated.

The degree of sedation, ${ }^{8,9,15}$ administration of muscle relaxants, ${ }^{9}$ intubation, ${ }^{3,8,15}$ method of bronchial toilet ${ }^{2}$ and duration of hospitalisation ${ }^{8}$ have all been implicated in the risk of ocular surface disease in the ICU patient. Our data agree with the relatively high correlation with the depth of sedation or use of muscle relaxants. However, in our study group the prevalence of keratopathy tended to decrease with duration of hospitalisation, being most prevalent in the first 2 weeks. This may be due to patients' stabilisation and weaning off sedation with consequent recovery of lid closure and the blink reflex.

Patients being nursed in the prone position to improve the ventilation perfusion ratio $(\mathrm{V} / \mathrm{Q})$ are at a high risk of exposure keratopathy. In our study population there were two patients who required this treatment. The first patient was noticed to develop severe exposure after $24 \mathrm{~h}$ of prone ventilation. The second patient had prophylactic lid taping during prone ventilation and remained free of keratopathy.
A survey of 20 ICUs in the United Kingdom carried out by Farrell and Wray ${ }^{16} 1993$ showed that the most common eye care protocol involves 2- to 6-hourly eye bathing with gauze soaked in normal saline or sterile water and the application of a polyacrylamide gel (Geliperm) to 'maintain' eyelid closure. Our own observations have shown that most patients do not need intervention to maintain eyelid closure and when they do the use of Geliperm may not achieve the desired effect. Lid closure with Micropore tape is widely advocated ${ }^{17}$ and has the advantage of maintaining good lid closure whilst allowing visualisation of the lid margins due to its transparency.

A counter-argument arises as to whether prolonged lid closure is itself detrimental to the ocular surface. It has been well illustrated by Baum ${ }^{18}$ that prolonged eyelid closure is a risk factor in the pathogenesis of several corneal conditions. Maurice ${ }^{19}$ proposed that REM sleep is a protective mechanism to stir up the aqueous humour to improve corneal oxygenation. ICU patients under sedation have reduced REM sleep, ${ }^{20,21}$ which may be a contributory factor.

The incidence of exposure keratopathy is closely related to heavy sedation or muscle relaxants and is highly prevalent in the first days of admission to ICUs. We now believe that the assessment of lid position in relation to the cornea is the single most important observation required in the ICU.

On the basis of a better understanding of the prevalence and of factors related to ocular surface damage in the ICU we have devised an algorithm that is currently being evaluated regarding its ease of use and ability to reduce the risk of ocular surface disease.

\section{References}

1. Hutton WL, Sexton RR. Atypical Pseudomonas corneal ulcers in semicomatose patients. Am J Ophthalmol 1972;73:37-9.

2. Hilton E, Adams AA, Uliss A, Lesser ML, Samuels S, Lowy FD. Nosocomial bacterial eye infections in intensive-care units. Lancet 1983;I:1318-20.

3. Ommeslag D, Colardyn F, Laey JJ. Eye infections caused by respiratory pathogens in mechanically ventilated patients. Crit Care Med 1987;13:80-1.

4. Wincek J, Ruttum MS. Exposure keratitis in comatose children. J Neurosci Nurs 1989;21:204-4.

5. Kirwan JF, Potamitis T, El-Kasaby H, Hope-Ross MW, Sutton GA. Microbial keratitis intensive care. BMJ 1997;314:433-4.

6. Baum J. Therapy for ocular bacterial infection. Trans Ophthalmol Soc UK 1986;105:69-77.

7. Parkin B, Turner A, Moore E, Cook S. Bacterial keratitis in the critically ill. Br J Ophthalmol 1997;81:1060-3.

8. Hernandez E, Mannis M. Superficial keratopathy in Intensive Care Unit patients. Am J Ophthalmol 1997;124:212-6.

9. Imanaka H, Taenaka N, Nakamura J, Aoyama K, Hosotani H. Ocular surface disorders in the critically ill. Anaesth Analg 1997;85:343-6.

10. Cook S, Palma O. Propofol as a sole agent for prolonged infusion in Intensive Care. J Drug Dev 1989;Suppl:65-7.

11. McClellan KA. Mucosal defense of the outer eye. Surv Ophthalmol 1997;42:233-46.

12. Moses RA. The eyelids. In: Moses RA, editor. Adler's physiology of the eye. St Louis: CV Mosby, 1981:15. 
13. Sachs R, Zagelbaum BM, Hersh PS. Corneal complications associated with the use of crack cocaine. Ophthalmology 1993;100:187-91.

14. Katz J, Kaufman HE. Corneal exposure during sleep (nocturnal lagophthalmos). Arch Ophthalmol 1977;95:449-53.

15. Lloyd F. Making sense of eye care for ventilated or unconscious patients. Nurs Times 1990;86:36-7.

16. Farrell M, Wray F. Eyecare for ventilated patients. Intensive Crit Care Nursing 1993;9:137-41.

17. Dua HS. Bacterial keratitis in the critically ill and comatose patient. Lancet 1998;351:387-8.
18. Baum JL. Prolonged eyelid closure is a risk to the cornea. Cornea 1997;16:602-11.

19. Maurice DM. The Von Sallmann Lecture 1996. An ophthalmological explanation of REM sleep. Exp Eye Res 1998;66:139-45.

20. Broughton R, Baron R. Sleep patterns in the intensive care unit and on the ward after acute myocardial infarction. Electroencephalogr Clin Neurophysiol 1978;45:348-60.

21. Schwab RJ. Disturbances of sleep in the intensive care unit. Crit Care Unit 1994;10:681-94. 